\title{
ON THE SINGULAR VECTORS OF THE GENERALIZED LYAPUNOV OPERATOR
}

\author{
Sheng Chen And Yunbo Tian
}

\begin{abstract}
In this paper, we study the largest and the smallest singular vectors of the generalized Lyapunov operator. For real matrices $A, B$ with order $n$, we prove that $\max _{\|X\|_{F}=1} \| A X B^{T}+$ $B X A^{T} \|_{F}$ is achieved by a symmetric matrix for $n \leqslant 3$ and give a counterexample for order $n=4$. We also prove that $\min _{\|X\|_{F}=1}\left\|A X B^{T}+B X A^{T}\right\|_{F}$ is achieved by a symmetric matrix for $n \leqslant 2$ and give a counterexample for order $n=3$. It is shown that the minimizer is symmetric, if the minimum is zero, or if the real parts of the eigenvalues of $A-\lambda B$ are of one sign.
\end{abstract}

Mathematics subject classification (2010): 65F35, 15A18, 15A45.

Keywords and phrases: Singular vectors, generalized Lyapunov operator, separation.

\section{REFERENCES}

[1] B. D. O. Anderson And J. B. Moore, Optimal Control-Linear Quadratic Methods, Prentice-Hall, Englewood Cliffs, NJ, 1990.

[2] R. H. Bartels and G. W. Stewart, Solution of the equation $A X+X B=C$, Comm. ACM 15 (1972), pp. 820-826.

[3] P. Benner And E. S. Quintana-OrTe, Solving stable generalized Lyapunov equations with the matrix sign function, Numerical Algorithms, 20 (1999), pp. 75-100.

[4] R. Bhatia AND D. DRISSI, Generalized Lyapunov equations and positive definite functions, SIAM Journal on Matrix Analysis and Applications, 27 (2005), pp. 103-114.

[5] R. Bhatia, Matrix analysis, Springer, 1997.

[6] R. BYERS, A LINPACK-style condition estimator for the equation $A X-X B^{T}=C$, IEEE Trans. Automat. Control., 29 (1984), pp. 926-928.

[7] R. BYERS AND S. NASH, On the singular "vectors" of the Lyapunov operator, SIAM J. Algebraic Discrete Methods, 8 (1987), pp. 59-66.

[8] R. BYERS AND D. KRESSNER, Structured condition numbers for invariant subspaces, SIAM Journal on Matrix Analysis and Applications, 28 (2006), pp. 326-347.

[9] S. Chen AND Y. Tian, Note on "On the singular "vectors" of the Lyapunov operator" by R. Byers and S. Nash, SIAM Journal on Matrix Analysis and Applications, 3 (2015), pp. 1069-1072.

[10] D. Cheng, On Lyapunov mapping and its applications, Communications in Information and Systems, 1 (2001), pp. 255-272.

[11] D. Cheng, Y. Zhu AND H. QI, A conjecture on the norm of Lyapunov mapping, Journal of Control Theory and Applications, 7 (2009), pp. 48-50.

[12] K.-W. E. CHU, The solution of the matrix equation $A X B-C X D=Y$ and $(Y A-D Z, Y C-B Z)=$ $(E, F)$, Linear Algebra Appl., 93 (1987), pp. 93-105.

[13] T. DAMM, Direct methods and ADI-preconditioned Krylov subspace methods for generalized Lyapunov equations, Numerical Linear Algebra with Applications, 15 (2008), pp. 853-871.

[14] J. D. Gardiner, A. J. Laub, J. J. Amato and C. B. Moler, Solution of the Sylvester matrix equation $A X B^{T}+C X D^{T}=E$, ACM Trans. Math. Software, 18 (1992), pp. 223-231.

[15] J. Feng, J. Lam, G. YAng And Z. LI, On a conjecture about the norm of Lyapunov mappings, Linear Algebra and its Applications, 465 (2015), pp. 88-103.

[16] S. J. HAMmARLING, Numerical solution of the stable non-negative definite Lyapunov equation, IMA J. Numer. Anal., 2 (1982), pp. 303-323. 
[17] U. Helmke and J. B. Moore, Optimization and Dynamical Systems, Springer, London, 1994.

[18] R. A. Horn And C. R. Johnson, Matrix analysis, Cambridge university press, 2012.

[19] B. Kagstrom And L. Westin, Generalized Schur methods with condition estimators for solving the generalized Sylvester equation, IEEE Trans. Automat. Control, 34 (1989), pp. 745-751.

[20] M. Konstantinov, V. Mehrmann and P. Petkov, On properties of Sylvester and Lyapunov operators, Linear Algebra Appl. 312 (2000), pp. 35-71.

[21] P. Lancaster And M. Tismenetsky, The Theory of Matrices, second ed., Academic Press, Orlando, FL, 1985.

[22] W. E. LongStafF, On tridiagonalization of matrices, Linear Algebra and its Applications, 109 (1988), pp. 153-163.

[23] V. Mehrmann, The Autonomous Linear Quadratic Control Problem, Theory and Numerical Solution, Lecture Notes in Control and Information Sciences, Vol. 163, Springer, Heidelberg, 1991.

[24] P. H. Petkov, N. D. Christov and M. M. Konstantinov, Computational Methods for Linear Control Systems, Prentice-Hall, Hertfordshire, UK, 1991.

[25] T. PENZL, Numerical solution of generalized Lyapunov equations, Advances in Computational Mathematics, 8 (1998), pp. 33-48.

[26] T. STYKeL, Stability and inertia theorems for generalized Lyapunov equations, Linear Algebra and its Applications, 355 (2002), pp. 297-314.

[27] W. Zhang, H. Zhang And B. S. Chen, Generalized Lyapunov equation approach to statedependent stochastic stabilization/detectability criterion, Automatic Control, IEEE Transactions on, 53 (2008), pp. 1630-1642.

[28] W. Zhang And B. S. Chen, Representation and Applications to Generalized Lyapunov Equations and Linear Stochastic Systems, Automatic Control, IEEE Transactions on, 57 (2012), pp. 3009-3022. 